\title{
Effects of Methoxyisoflavone, Ecdysterone, and Sulfo-Polysaccharide Supplementation on Training Adaptations in Resistance-Trained Males
}

\author{
Colin D. Wilborn ${ }^{1}$, Lemuel W. Taylor ${ }^{2}$, Bill I. Campbell ${ }^{3}$, Chad Kerksick ${ }^{4}$, Chris J. Rasmussen ${ }^{3}$, \\ Michael Greenwood ${ }^{3}$, Richard B. Kreider ${ }^{3}$
}

Human Performance Lab, University of Mary Hardin Baylor, Belton, TX ${ }^{1}$; Exercise Physiology Lab, University of West Florida, Pensacola, $\mathrm{FL}^{2}$; Exercise and Sport Nutrition Laboratory, Baylor University, Waco, TX ${ }^{3}$; Applied Biochemistry and Molecular Physiology Laboratory, University of Oklahoma, Norman, $\mathrm{OK}^{4}$. Address correspondence to richard_kreider@baylor.edu.

Recieved September 8, 2006/Accepted November 16, 2006

\section{ABSTRACT}

PURPOSE: Methoxyisoflavone (M), 20-hydroxyecdysone (E), and sulfo-polysaccharide (CSP3) have been marketed to athletes as dietary supplements that can increase strength and muscle mass during resistancetraining. However, little is known about their potential ergogenic value. The purpose of this study was to determine whether these supplements affect training adaptations and/or markers of muscle anabolism/catabolism in resistance-trained athletes. METHODS: Forty-five resistance-trained males $(20.5 \pm 3$ yrs; $179 \pm 7 \mathrm{~cm}, 84 \pm 16 \mathrm{~kg}, 17.3 \pm 9 \%$ body fat) were matched according to FFM and randomly assigned to ingest in a double blind manner supplements containing either a placebo (P); $800 \mathrm{mg} / \mathrm{day}$ of M; $200 \mathrm{mg}$ of E; or, 1,000 $\mathrm{mg}$ /day of CSP3 for 8-weeks during training. At 0, 4, and 8-weeks, subjects donated fasting blood samples and completed comprehensive muscular strength, muscular endurance, anaerobic capacity, and body composition analysis. Data were analyzed by repeated measures ANOVA. RESULTS: No significant differences ( $>0.05)$ were observed in training adaptations among groups in the variables FFM, percent body fat, bench press 1RM, leg press $1 \mathrm{RM}$ or sprint peak power. Anabolic/catabolic analysis revealed no significant differences among groups in active testosterone (AT), free testosterone (FT), cortisol, the AT to cortisol ratio, urea nitrogen, creatinine, the blood urea nitrogen to creatinine ratio. In addition, no significant differences were seen from pre to post supplementation and/or training in AT, FT, or cortisol. CONCLUSION: Results indicate that M, E, and CSP3 supplementation do not affect body composition or training adaptations nor do they influence the anabolic/catabolic hormone status or general markers of catabolism in resistance-trained males. Journal of the International Society of Sports Nutrition. 3(2): 19-27, 2006

Key Words: resistance training, sports nutrition, supplementation, exercise, anabolic

\section{INTRODUCTION}

Numerous nutritional supplements are marketed as ergogenic aids to resistance-trained athletes in an attempt to promote increases in strength, power, and/or muscle mass during training. Over the last few years, methoxyisoflavone, ecdysterones, and sulfo-polysaccharide (a purported myostatin inhibitor) have been marketed as potential anabolic promoting nutritional supplements for resistancetrained athletes. Unfortunately, little well-controlled research has been conducted to examine the potential ergogenic properties of these nutrients.
Methoxyisoflavone is a member of the flavonoids (isoflavones) family that are primarily obtained in the diet from soybeans and soy foods ${ }^{1}$. The two most popular forms of methoxyisoflavones on the market are 5-methyl-7-methoxy-isoflavone (Methoxyisoflavone) and 7-Isopropoxyisoflavone (Ipriflavone). Initial claims based on animal research suggested that methoxyisoflavone supplementation possesses a muscle-building and bone-building (anabolic) component without the side effects of traditional hormone replacement therapies that would give similar results ${ }^{1}$. Despite these claims, the only beneficial effect of methoxyisoflavone that has been reported in multiple research publications and U.S. 
patents is the benefits of reduced bone resorption and bone loss prevention ${ }^{2-4}$.

Ecdysterone (20-Beta-Hydroxyecdysterone) is a plant sterol that has also been linked to some bold claims including promotion of protein synthesis, maintenance of anabolic state, and enhancement of lean muscle mass, while subsequently decreasing adipose tissue. Some common names for ecdysterone include ecdisten, ecdysone, isoinokosterone, 20-hdroxyecdysone and Becdysterone. Currently the only research supporting these claims has been conducted in animals where reports suggest ecdysterone's lead to anabolic activity on skeletal muscles ${ }^{5}$, cell proliferation and growth leading to increased mass from vitamin-like effects ${ }^{6}$, improved liver secretory function ${ }^{7}$ as they play a structural role in the mitochondrial membranes in cells ${ }^{8}$, as well as immunomodulating effects ${ }^{9}$. Despite these potential benefits, no research has supported these claims in human models.

Sulfo-polysaccharide is a nutrient that is advertised to bind to myostatin and inhibit its activity in muscle. Sulfo-polysaccharide's active ingredient is a brown sea algae known as cystoseira canariensis. Myostatin is a cytokine that works by inhibiting the proliferation of satellite cells and the differentiation of myoblasts ${ }^{10}$, while also decreasing adipogenesis via reductions in the secretion of leptin ${ }^{11}$. Thus, the rationale for binding myostatin with sulfopolysaccharides would theoretically result in increased muscle mass and improved body composition. The interest in myostatin was generated by recent studies where antibodies for myostatin were created and administered to adult rodents, which resulted in an increase in body mass, muscle mass, muscle size, and strength ${ }^{11,12}$. Despite the success in rodents, human models have not been as successful in increasing body mass and muscle mass ${ }^{13}$, but sulfo-polysaccharides have been reported to have a binding specificity for the myostatin inhibitor follistatin ${ }^{14}$. Further research is needed to see if sulfo-polysaccharides or cystoseira canariensis have any beneficial effect in humans in response to resistance training.

Despite profound claims, little is known regarding the effects of dietary supplementation of these nutrients on training adaptations in resistance-trained subjects. Therefore, the purpose of this study was to examine the effects of methoxyisoflavone, ecdysterone, and sulfo-polysaccharide (CSP3) supplementation during resistance training on body composition, markers of catabolism, and training adaptations.

\section{METHODOLOGY}

Subjects. Forty-five resistance-trained males who had been consistently training for at least one year volunteered for this study. Subjects were considered resistance trained if they had been consistently training for one year, a minimum of three days per week. Subjects were with $20.5 \pm 3$ yrs, $179 \pm 7 \mathrm{~cm}$, $84 \pm 16 \mathrm{~kg}$, and $17.3 \pm 9 \%$ body fat. Subjects signed an informed consent that was approved by the Institutional Review Board for Human subjects prior to participation. Each subject completed a personal information sheet and a standard medical history form verified by a registered nurse. Subjects were free from any major metabolic disorders (i.e. heart disease, diabetes, thyroid disease, etc.) as well as major musculoskeletal disorders that would interfere with their ability to workout and/or complete the tests during the three testing sessions. Subjects were not permitted in the study if they had taken any selfreported ergogenic dietary supplements (i.e. creatine, androstendione, myostatin inhibitors, pro-hormones, etc.) within six months prior to the onset of the study. Subjects were asked to maintain their normal diet throughout the study and were not allowed to ingest any dietary supplement that contained potentially ergogenic nutrients. However, subjects were permitted to ingest energy-based sports drinks, energy bars, and protein powders provided that they did not contain any ergogenic nutrients. The reason for this was that many resistance-trained athletes ingest these energy-based supplements as a means to maintain their recommended dietary intake of calories. This intake was considered as part of their normal diet and was accounted for in analysis of dietary intake.

Experimental Design. The study was conducted as a double-blind, placebo controlled trial using parallel groups matched according to fat free mass. The independent variable was nutritional supplementation. Dependent variables included: dietary intake, body mass, body water (total, intracellular, extracellular) using bioelectrical impedance, body composition using dual energy $x$ ray absorptiometry (DEXA), upper and lower body 1 RM strength (bench press and leg press), muscle endurance $(80 \%$ of $1 \mathrm{RM}$ on bench press and leg press), anaerobic sprint power (Wingate cycle ergometer test), fasting clinical blood profiles (substrates, electrolytes, muscle and liver enzymes, 
red cells, white cells), and anabolic/catabolic hormones (free and active testosterone, cortisol).

Familiarization and Testing Sessions. Subjects were pre-qualified for entry into the study and then familiarized to the experimental design and practiced the exercise tests in order to get acquainted with the nature of the equipment and protocol prior to baseline testing. Subjects were scheduled for their first testing session and all questions and concerns were answered at this time.

Prior to baseline testing (T1), subjects recorded dietary intake on diet record forms for 4-days (three weekdays and one weekend day). Dietary records were analyzed by a registered dietitian using ESHA Research Inc. (Salem, OR) nutritional software. Subjects were instructed to abstain from exercise or physical activity for 48 hours prior to testing and were instructed to fast at least 10 hours prior to the baseline blood draw. On the day of baseline testing, subjects reported to the lab and completed questionnaires and information sheets. Height was measured using standard anthropometry and total body weight was measured using a calibrated electronic scale with a precision of $\pm 0.02 \mathrm{~kg}$ (HealthO-Meter, Bridgeview, IL). Whole-body composition was estimated by certified personnel using a Hologic QDR-4500W dual-energy x-ray absorptiometry (DEXA) using Hologic software version 9.80C (Waltham, MA). This test evaluates body composition and body density by scanning the entire body with a low dose of radiation. Test-retest reliability studies performed on male athletes with this DEXA machine yielded a mean deviation for total bone mineral content and total fat free/soft tissue mass of $0.31 \%$ with a mean intra-class correlation of 0.985 . This method of determining body composition has been shown to be valid ${ }^{15}$.

Following the DEXA, subjects donated approximately $20 \mathrm{ml}$ of fasting blood from the antecubital vein in the arm via venipuncture using standard and sterile procedures. Blood was analyzed for basic clinical chemistry profiles for safety measures [glucose, protein, blood urea nitrogen (BUN), creatinine, uric acid, aspartate aminotransferase (AST), alanine aminotransferase (ALT), creatine kinase (CK), lactate dehydrogenase (LDH), gamma glutamyl transaminase (GGT), triglycerides, cholesterol] and whole blood cell counts [including hemoglobin, hematocrit, red blood cell counts, mean corpuscle volume (MCV), mean corpuscle hemoglobin $(\mathrm{MCH})$, mean corpuscle hemoglobin concentration (MCHC), red cell dimension width (RDW), white blood cells (WBC), neutrophils, lymphocytes, monocytes, basophils, and eosinophils] by Quest Diagnostics (Dallas, TX). Blood serum samples were stored for later analysis of the anabolic/catabolic hormones (active testosterone, free testosterone, and cortisol) via assays in the Exercise and Biochemical Nutrition Laboratory.

Subjects first warmed-up ( 2 sets of $8-10$ repetitions at approximately $50 \%$ of anticipated maximum) on the bench press. Subject's then performed successive 1-RM lifts starting at about $70 \%$ of anticipated 1-RM and increasing by $5-10 \mathrm{lbs}$ until the subject reached their 1-RM. After the acquisition of $\max$ the participant rested five minutes and completed as many repetitions as possible at $80 \% 1-\mathrm{RM}$ to assess muscular endurance. Subjects were instructed on proper technique and mechanics of the movement. Hand position was also recorded to ensure test re-test reliability. Subjects then rested for 10 minutes and warmed-up on the Nebula $45^{\circ}$ Leg press ( 2 sets of $8-$ 10 repetitions at approximately $50 \%$ of anticipated maximum). Subjects then performed successive 1 RM lifts on the leg press starting at about $70 \%$ of anticipated 1RM and increasing by $10-25 \mathrm{lbs}$ until reaching the subject's $1 \mathrm{RM}$. Subjects then perform an $80 \%$ of $1 \mathrm{RM}$ endurance repetition tests on the hip/leg sled. Foot placement and sled height were recorded to ensure test re-test reliability. All strength testing was supervised by a Certified Strength and Conditioning Specialist (CSCS). Test re-test reliability of performing these strength tests on resistance-trained subjects in our laboratory have yielded low mean coefficients of variation and high reliability for the bench press $(1.9 \%$, intraclass $r=$ $0.94)$ and leg press/hip sled $(0.7 \%$, intraclass $r=$ $0.91)$.

Following the strength assessments and 10 minutes of rest, subjects then perform a 30-second Wingate anaerobic capacity test using a Lode computerized cycle ergometer (Groningen, Netherlands). Correlation coefficients of test-retest reliability for absolute peak power and mean power in our lab has been found to be $r=0.69$ and $r=0.95$, respectively. Subjects replicated all testing after 4 and 8 weeks of training and supplementation.

Supplementation Protocol. Subjects were matched into one of four groups according to fat free mass. Subjects were then randomly assigned to ingest in a double blind manner capsules containing a dextrose placebo (P); $800 \mathrm{mg}$ /day of methoxyisoflavone (M) (MuscleTech Research \& Development, Inc., Mississauga, ON); $100 \mathrm{mg} /$ day of Polypodium 
Vulare/Suma root standardized for $30 \mathrm{mg}$ of 20 hydroxyecdysone $(\mathrm{E})$; or, $500 \mathrm{mg} /$ day of sulfopolysaccharides (CSP3) extracted from Cystoseira canariensis (Biotest Labs, Colorado Springs, CO). Subjects ingested the assigned capsules in the morning once per day for 8-weeks. The supplements were prepared in capsule form and packaged in generic bottles for double blind administration by MuscleTech Research \& Development, Inc., (Mississanga, ON). Supplementation compliance was monitored by research assistants by having the subjects return empty bottles of the supplement at the end of 4 and 8 weeks of supplementation.

Training Protocol. Subjects participated in a periodized 4-day per week resistance-training program split into two upper and two lower extremity workouts per week for a total of 8-weeks. Prior to the workout, subjects performed a standardized series of stretching exercises as a warm-up. Subjects then performed an upper body resistance-training program consisting of nine exercises (bench press, lat pull, shoulder press, seated rows, shoulder shrugs, chest flys, biceps curl, triceps press down, and abdominal curls) twice per week. Subjects also performed a seven-exercise lower extremity resistance-training program that could include (leg press, squat, back extension, step-ups, leg curls, leg extension, heel raises, and abdominal crunches) twice per week. The program was standardized at 3 sets of 10 repetitions with as much weight as they could lift per set (typically $60-80 \%$ of $1 \mathrm{RM}$ ) with no more than 2 minute rest periods between sets and no more than 3 minutes of rest between exercises. All training was conducted at the Student Life Center (SLC) at Baylor University. Subjects recorded the amount of weight lifted and number of repetitions performed for each set on training cards so that training volume could be determined. Subjects were also instructed to have their exercise card signed by SLC staff in order to verify attendance and completion of the workouts.

Blood and Serum Analysis. Two serum separation vacutainer tubes and one EDTA vacutainer tube was obtained from each subject. The serum vacutainers were separated via centrifugation a $6,000 \mathrm{rpm}$ for 20 minutes. One serum separation tube and the EDTA tube were sent to Quest Diagnostics (Dallas, TX) for assay of a standard clinical chemistry profile and whole blood cell counts to ensure safety of supplementation during the protocol. The serum from the remaining separation tube was separated, labeled, and stored in micro centrifuge tubes at $-80^{\circ} \mathrm{C}$ for later analysis. Following completion of the study, samples were analyzed for active testosterone, free testosterone, and cortisol with an enzyme immunoassay (EIA) assays using Goat-Anti-rabbit IgG (GARG) coated microplates. EIA kits were purchased from Diagnostic Systems Laboratories (Webster, TX). Assays were performed using a Jitterbug microplate shaker (Boekel ScientificPhiladelphia, $P A$ ) and a Tricontinent Multiwash Advantage microplate washer (Grass Valley, CA). The assays were run in duplicate and the absorbances of the standards, samples, and controls were determined at an optical density of 450 nanometers with a Wallac Victor $^{2} 1420$ Multilabel counter by PerkinElmer (Boston, MA). Concentrations of active testosterone, free testosterone, and cortisol were expressed relative to changes in blood serum content. Intra-assay coefficients of variation were $5.3 \%$ and $6.8 \%, 7.5 \%$ and $5.4 \%$, and $2.4 \%$ and $5.0 \%$, respectively, for active testosterone (control I and II), free testosterone (control I and II), and cortisol (control I and II). Inter-assay coefficients of variation were $4.8 \%$ and $4.9 \%, 0.22 \%$ and $1.28 \%$, and $12.0 \%$ and $6.1 \%$, respectively, for active testosterone (control I and II), free testosterone (control I and II), and cortisol (control I and II).

Data Analysis. Data were analyzed using SPSS 11.5 for Windows (Chicago, Illinois). A repeated measures analysis of variances (ANOVA) was used for the analysis of the univariate means on the dependent variables and is presented in means and standard deviations. Delta scores (post and pre values) were calculated on selected variables and analyzed by ANOVA for repeated measures. Date were considered significant when the probability of Type I error was 0.05 or less. Significant group $\mathrm{x}$ time interactions were evaluated by least significant differences (LSD) post-hoc analysis to determine where the significance existed. Power analysis in a 4 x 3 design indicate that an n-size of 15 per group yields high power $(>0.9)$ for delta values of 0.75 to 1.25. Data are presented as means $\pm \mathrm{SD}$ changes from baseline.

\section{RESULTS}

Side Effects. Analysis of post study questionnaires revealed that subjects tolerated the supplementation protocol well with no reports of medical problems or symptoms.

Training Volume and Dietary Intake. No statistically significant differences were observed among groups in total lifting volume during the training period for upper body (P 322,984 $\pm 106,936$; M 304,987 $\pm 51,955$; E 275,744 $\pm 72,658$; CSP3 
$313,308 \pm 112,077 \mathrm{~kg} ; \mathrm{p}=0.83)$ and lower body $(\mathrm{P}$ $398,976 \pm 77,553 ;$ M 408,966 $\pm 113,746 ;$ E 448,371 $\pm 189,738$; CSP3 422,163 $\pm 167,259 \mathrm{~kg} ; \mathrm{p}=0.94$ ). Table 1 presents caloric intake data observed among groups throughout the trial. No significant group or group $\mathrm{x}$ time effects were observed in mean daily total caloric carbohydrate, protein, or fat intake.

Body Mass and Body Composition. Figure 1 presents mean changes in body mass and body composition for the $\mathrm{P}, \mathrm{M}, \mathrm{E}$, and CSP3 groups. Training resulted in a statistically significant increase $(p=0.03)$ in DEXA fat free mass (lean/soft tissue mass plus bone mineral content) for all groups. However, no statistically significant differences were observed among the P, M, E, and CSP3 groups, respectively, in mean fat free mass $(\mathrm{P} 0.94 \pm 1.5$; M $0.29 \pm 1.4$; E $0.04 \pm 1.3 ;$ CSP3 $0.64 \pm 1.4 \mathrm{~kg}, \mathrm{p}=0.40$ ), fat mass $(\mathrm{P} 0.69 \pm 1.1 ; \mathrm{M} 0.32 \pm 1.6 ;$ E $0.69 \pm 1.1$; CSP3 $0.36 \pm 0.7 \mathrm{~kg}, \mathrm{p}=0.17$ ), percent body fat ( $\mathrm{P} 0.59 \pm$ 1.7 ; M $0.08 \pm 1.7$; E $0.70 \pm 1.0$; CSP3 $-0.46 \pm 0.8 \%$, $\mathrm{p}=0.18)$, or total body water (P $0.39 \pm 3.3$, M $0.16 \pm$ 2.9, E $1.7 \pm 2.5, \mathrm{CSP} 30.31 \pm 2.4 \mathrm{~L}, \mathrm{p}=0.61$ ).

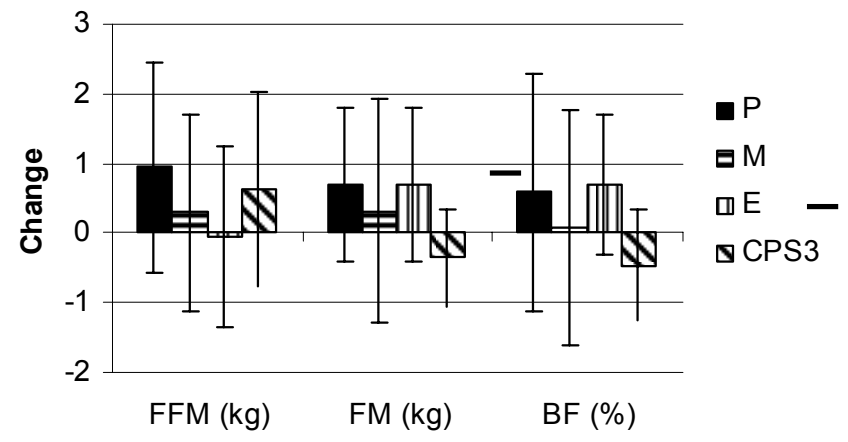

Figure 1. Change in body composition variables (mean $\pm \mathrm{SD})$ fat free mass (FFM), fat mass (FM) and body fat (BF) between groups following 8 weeks of resistance training and supplementation.

Training Adaptations. Training resulted in statistically significant increase in 1-RM bench press (pre: $94.94 \pm 22.8$ to post: $100.84 \pm 20.54 \mathrm{~kg}, \mathrm{p}=$ $<0.0001)$ and $1-\mathrm{RM}$ leg press $(311 \pm 76$ to $341 \pm 82$ $\mathrm{kg}, \mathrm{p}=<0.0001$ ) for all groups. No statistically significant interactions were observed among $\mathrm{P}, \mathrm{M}$, $\mathrm{E}$, and CSP3 groups, respectively, in mean changes in bench press $1-\mathrm{RM}(6.2 \pm 7 ; 2.5 \pm 5 ; 7.2 \pm 10 ; 7.7 \pm 6$ $\mathrm{kg}, \mathrm{p}=0.29)$, leg press $1-\mathrm{RM}(20.6 \pm 33 ; 37.0 \pm 39$; $38.9 \pm 45 ; 29.3 \pm 36 \mathrm{~kg}, \mathrm{p}=0.63)$. Bench press lifting volume was significantly greater in the $\mathrm{M}$ group $(105$ $\pm 120 ; 102 \pm 200 ; 124 \pm 174 ; 25 \pm 176 \mathrm{~kg}, \mathrm{p}=0.008)$ while leg press lifting volume was lower in the M group $(976 \pm 1,213 ;-799 \pm 1,718 ; 1,259 \pm 1,153 ; 978$ $\pm 2,328 \mathrm{~kg}, \mathrm{p}=0.04)$. Likewise, no significant differences were observed in mean changes in Wingate peak power $(82 \pm 248 ; 30 \pm 139 ; 28 \pm 151$; $17 \pm 171 \mathrm{~W}, \mathrm{p}=0.82)$, Wingate mean power $(20 \pm 83$; $-13 \pm 88 ;-18 \pm 76 ;-15 \pm 52 \mathrm{~W}, \mathrm{p}=0.55)$, or Wingate total work $(582 \pm 2,564 ;-216 \pm 2,716 ;-420 \pm 2,291$; $-528 \pm 1,514 \mathrm{~J}, \mathrm{p}=0.29)$.

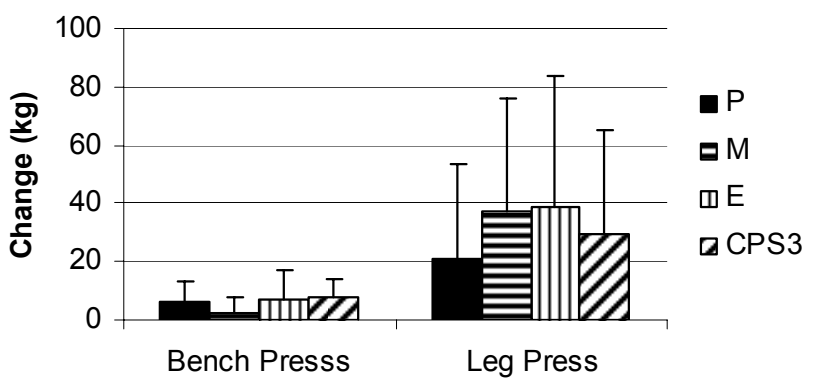

Figure 2. Change in bench press and leg press 1-repetition maximum levels (mean $\pm \mathrm{SD}$ ) between groups following 8 weeks of resistance training and supplementation.

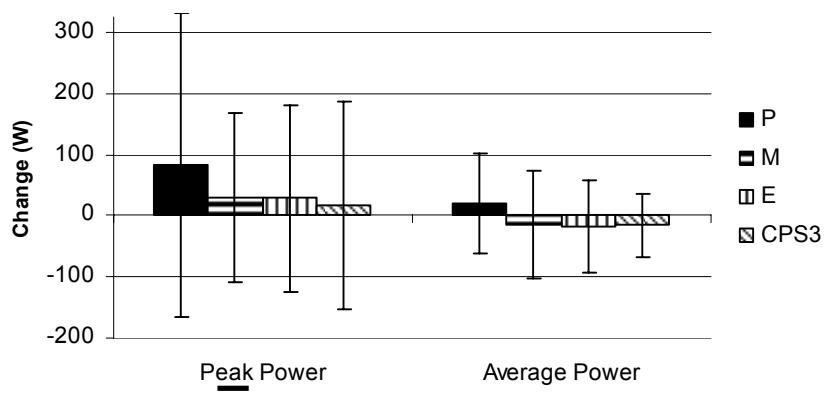

Figure 3. Change in Wingate sprint performance (mean \pm SD) between groups following 8 weeks of resistance training and supplementation.

Blood Chemistry Profiles. No statistically significant differences were observed among groups as well as over time for the clinical chemistry profiles on variables including serum protein $(\mathrm{p}=0.65)$, uric acid $(\mathrm{p}=0.57)$, AST $(\mathrm{p}=0.16), \operatorname{ALT}(\mathrm{p}=0.56)$, GGT $(p=0.86)$, triglycerides $(p=0.62)$, and total cholesterol $(\mathrm{p}=0.48)$. No statistically significant differences were observed among groups as well as over time in the whole blood cell count variables including hemoglobin $(\mathrm{p}=0.74)$, hematocrit $(\mathrm{p}=$ $0.61)$, red blood cell counts $(\mathrm{p}=0.39), \mathrm{MCV}(\mathrm{p}=$ $0.68), \mathrm{MCH}(\mathrm{p}=0.20), \operatorname{MCHC}(\mathrm{p}=0.29), \mathrm{WBC}(\mathrm{p}=$ $0.076)$, lymphocytes $(p=0.44)$, monocytes $(p=$ $0.66)$, and eosinophils $(\mathrm{p}=0.28)$ from whole blood analysis. However, significant changes were seen in neutrophils $(2.05 \pm 9.9 ; 4.4 \pm 10.1 ; 4.3 \pm 5.5,5.8 \pm$ $7.4 \mathrm{k} / \mu \mathrm{L}:, \mathrm{p}=0.04)$ and basophils $(7.5 \pm 7.6 ; 7.6 \pm$ $25.1 ; 6.57 \pm 16.1 ; 4.4 \pm 7.8 \mathrm{k} / \mu \mathrm{L}, \mathrm{p}=0.04) . \quad$ All values remained within normal clinical ranges. 
Table 1. Dietary intake data for the $\mathrm{P}, \mathrm{M}, \mathrm{E}$, and CSP3 groups.

\begin{tabular}{|c|c|c|c|c|c|}
\hline Variable/Group & $\mathrm{T} 1$ & $\mathrm{~T} 2$ & $\mathrm{~T} 3$ & Signif & nce \\
\hline \multicolumn{6}{|l|}{$\operatorname{Protein}(\mathrm{g} / \mathrm{kg} / \mathrm{d})$} \\
\hline $\mathrm{P}$ & $1.6 \pm .7$ & $1.7 \pm .85$ & $1.5 \pm .6$ & Group & .577 \\
\hline M & $1.5 \pm .68$ & $1.4 \pm .53$ & $1.4 \pm .52$ & Time & .068 \\
\hline $\mathrm{E}$ & $1.7 \pm .34$ & $1.7 \pm .34$ & $1.4 \pm .44$ & GxT & .815 \\
\hline $\mathrm{CSP} 3$ & $1.4 \pm .41$ & $1.4 \pm .41$ & $1.3 \pm .59$ & & \\
\hline \multicolumn{6}{|l|}{ Fat $(\mathrm{g} / \mathrm{kg} / \mathrm{d})$} \\
\hline $\mathrm{P}$ & $1.7 \pm .5$ & $1.5 \pm .5$ & $1.3 \pm .8$ & Group & .866 \\
\hline M & $1.7 \pm 1.6$ & $1.3 \pm .6$ & $1.2 \pm .49$ & Time & .092 \\
\hline $\mathrm{E}$ & $1.5 \pm .8$ & $1.2 \pm .29$ & $1.2 \pm .39$ & GxT & .670 \\
\hline CSP3 & $1.1 \pm 1.3$ & $1.5 \pm 1.3$ & $1.0 \pm .42$ & & \\
\hline \multicolumn{6}{|c|}{ Carbohydrates $(\mathrm{g} / \mathrm{kg} / \mathrm{d})$} \\
\hline $\mathrm{P}$ & $5.5 \pm 3.5$ & $4.6 \pm 1.4$ & $4.2 \pm 1.3$ & Group & .293 \\
\hline M & $3.5 \pm 1.7$ & $3.5 \pm 1.7$ & $4.0 \pm 2.3$ & Time & .001 \\
\hline $\mathrm{E}$ & $4.7 \pm 1$ & $3.8 \pm .83$ & $3.9 \pm 1.2$ & GxT & .491 \\
\hline CSP3 & $3.8 \pm 1.7$ & $3.4 \pm 1.6$ & $3.4 \pm 1.7$ & & \\
\hline \multicolumn{6}{|c|}{ Total Calories $(\mathrm{kcal} / \mathrm{kg} / \mathrm{d})$} \\
\hline $\mathrm{P}$ & $40 \pm 10$ & $39 \pm 11$ & $41 \pm 37$ & Group & .331 \\
\hline M & $40 \pm 28$ & $31 \pm 13$ & $32 \pm 15$ & Time & .274 \\
\hline E & $40 \pm 12$ & $31 \pm 6$ & $32 \pm 7$ & GxT & .826 \\
\hline CSP3 & $30 \pm 12$ & $29 \pm 13$ & $28 \pm 11$ & & \\
\hline
\end{tabular}

Data are means and \pm standard deviations

Table2. Selected markers of catabolism for the P, M, E, and CSP3 groups.

\begin{tabular}{|c|c|c|c|c|c|}
\hline Variable/Group & $\mathrm{T} 1$ & $\mathrm{~T} 2$ & $\mathrm{~T} 3$ & Signif & nce \\
\hline \multicolumn{6}{|l|}{ Creatinine $(\mathrm{mg} / \mathrm{dl})$} \\
\hline $\mathrm{P}$ & $1.15 \pm .11$ & $1.14 \pm .14$ & $1.2 \pm .1$ & Group & .812 \\
\hline M & $1.2 \pm .14$ & $1.2 \pm .13$ & $1.2 \pm .13$ & Time & .891 \\
\hline $\mathrm{E}$ & $1.2 \pm .13$ & $1.1 \pm .16$ & $1.2 \pm .13$ & GxT & .537 \\
\hline CSP3 & $1.1 \pm .17$ & $1.1 \pm .15$ & $1.1 \pm .12$ & & \\
\hline \multicolumn{6}{|l|}{ BUN (mg/dl) } \\
\hline $\mathrm{P}$ & $16.9 \pm 3.3$ & $14.4 \pm 3.7$ & $16 \pm$ & Group & .959 \\
\hline $\mathrm{M}$ & $16.1 \pm 3.5$ & $14.9 \pm 3.5$ & $15.1 \pm \quad 4.4$ & Time & .041 \\
\hline $\mathrm{E}$ & $16.2 \pm 3.1$ & $15 \pm 2.7$ & $14.7 \pm \quad 3.3$ & GxT & .970 \\
\hline CSP3 & $16.3 \pm 2.6$ & $14.8 \pm 3.5$ & $15.1 \pm \quad 2.6$ & & \\
\hline \multicolumn{6}{|l|}{ CK (IU/L) } \\
\hline $\mathrm{P}$ & $207 \pm 1088$ & $652 \pm 1556$ & $250 \pm 188$ & Group & .644 \\
\hline M & $198 \pm 98$ & $171 \pm 782$ & $45 \pm 188$ & Time & .383 \\
\hline $\mathrm{E}$ & $172 \pm 83$ & $228 \pm 133$ & $159 \pm 64$ & GxT & .091 \\
\hline CSP3 & $594 \pm 108$ & $174 \pm 90$ & $346 \pm 614$ & & \\
\hline \multicolumn{6}{|l|}{ LDH (IU/L) } \\
\hline $\mathrm{P}$ & $132 \pm 15$ & $143 \pm 36$ & $135 \pm 24$ & Group & .114 \\
\hline M & $139 \pm 16$ & $138 \pm 22$ & $150 \pm 22$ & Time & .752 \\
\hline $\mathrm{E}$ & $126 \pm 23$ & $124 \pm 12$ & $124 \pm 14$ & GxT & .359 \\
\hline CSP3 & $150 \pm 33$ & $137 \pm 14$ & $141 \pm 17$ & & \\
\hline \multicolumn{6}{|l|}{ Cortisol $(\mu \mathrm{g} / \mathrm{dl})$} \\
\hline $\mathrm{P}$ & $16.5 \pm 5.7$ & $14.6 \pm 5.2$ & $13.8 \pm 3.5$ & Group & .706 \\
\hline M & $13.2 \pm 4.3$ & $13.2 \pm 4.3$ & $15.5 \pm 5.1$ & Time & .222 \\
\hline $\mathrm{E}$ & $17.1 \pm 4.5$ & $15.6 \pm 5.3$ & $15.1 \pm 5.8$ & GxT & .571 \\
\hline CSP3 & $16.7 \pm 9.5$ & $17.5 \pm 5.7$ & $15.0 \pm 5.6$ & & \\
\hline
\end{tabular}

Data are means and \pm standard deviations 
Anabolic/Catabolic Status. Table 2 presents selected markers of anabolic and catabolic status while Figure $4 \& 5$ show changes in free and active testosterone for the P, M, E, and CSP3 groups. No statistically significant differences were observed among P, M, E, and CSP3 groups, respectively, in changes in total testosterone $(0.21 \pm 0.83,0.26 \pm 1,0.02 \pm 1.21,0.10 \pm$ $0.93 \mathrm{ng} / \mathrm{ml}, \mathrm{p}=0.67)$, free testosterone $(4.39 \pm 10.4$, $5.54 \pm 6.85,1.39 \pm 11.84,1.77 \pm 8.62 \mathrm{pg} / \mathrm{ml}, \mathrm{p}=$ $0.25)$, cortisol $(2.68 \pm 2.46,1.11 \pm 6.3,1.93 \pm 5.57$, $1.66 \pm 10.54 \mu \mathrm{g} / \mathrm{dl}, \mathrm{p}=0.57)$, total testosterone to cortisol ratio $(0.05 \pm 0.09,0.02 \pm 0.16,0.06 \pm 0.17$, $0.02 \pm 0.16, p=0.43)$. In addition, no differences were observed from pre to post testing for all variables in Table 2.

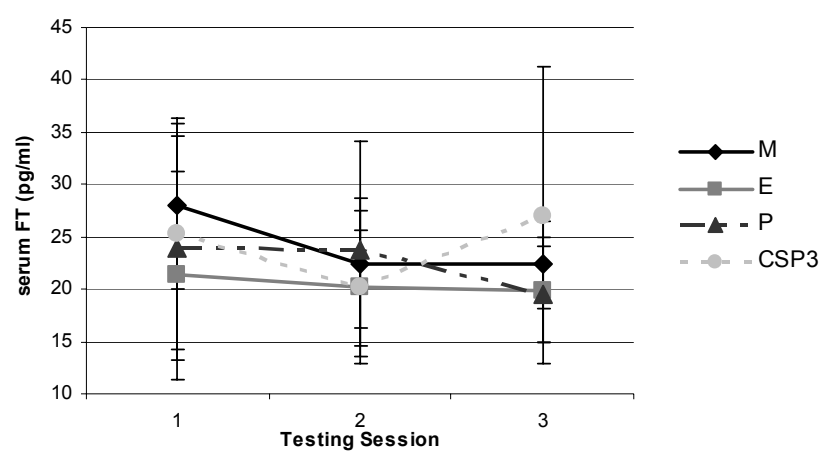

Figure 4. Change in serum free testosterone levels (mean \pm SD) between groups following 8 weeks of resistance training and supplementation.

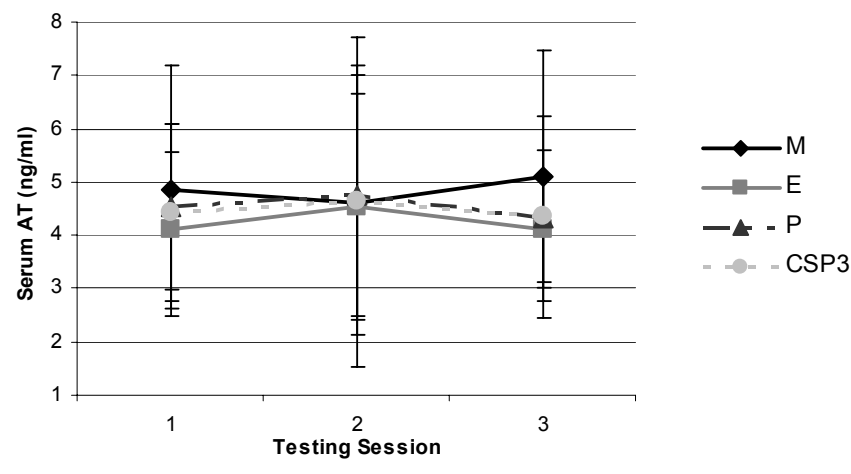

Figure 5. Change in serum active testosterone levels (mean \pm SD) between groups following 8 weeks of resistance training and supplementation.

\section{DISCUSSION}

The purpose of this study was to determine whether methoxyisoflavone, 20-hydroxyecdysone, or sulfopolysaccharide supplementation affects muscle mass, training adaptations, or markers of muscle growth and/or breakdown in resistance-trained males. The major finding of this study was that dietary supplementation of commercially available supplements containing methoxyisoflavone, ecdysterone, and sulfopolysaccharide (CSP3) did not significantly affect anabolic or catabolic responses to resistance training, body composition, or training adaptations. These findings do not support contentions that methoxyisoflavone, ecdysterone, and sulfopolysaccharide (CSP3) supplementation during resistance training enhance gains in strength, power or muscle mass.

Isoflavones are naturally occurring non-steroidal phytoestrogens found primarily in soy beans ${ }^{1}$. 5methyl-7-methoxy-isoflavone is believed to play a role in increased protein synthesis and muscle accretion. They are also believed to reduce body fat, lower cholesterol levels, promote endurance, increase vitality, and the body's ability to use oxygen. The primary foundation of these beliefs have been data described in a U.S. patent in the early 1970's ${ }^{16,17}$. Feurer et al ${ }^{16}, 17$ reported lower cortisol levels, increased protein synthesis, and improved overall recovery from exercise as a result of isoflavone supplementation in animals. Preliminary results from a study only available in abstract form ${ }^{18}$ evaluated the effects of 5-methyl-7-methoxyisoflavone supplementation ( $800 \mathrm{mg} /$ day for 8 -weeks) on training adaptations in 14 resistance-trained men. Inclendon et $\mathrm{al}^{18}$ reported 5-methyl-7methoxyisoflavone supplementation did not significantly affect changes in body weight, body mass index, bone mineral content, or isokinetic peak force between groups. However, DEXA determined FFM increased by $1.3 \mathrm{~kg}$ in the methoxyisoflavone group while being unchanged $(0.1 \mathrm{~kg})$ in the placebo group resulting in a significant reduction in body fat percent. Results of the present study do not support the purported ergogenic value of 5-methyl-7methoxyisoflavone supplementation in resistancetrained males.

Ecdysterones have also been recently purported to enhance training adaptations during resistance training. In support of this contention, research in animal models has suggested that ecdysterone supplementation can promote anabolic activity in skeletal muscle ${ }^{5}$, as well as increase cell proliferation and growth, which can lead to an increase in muscle mass ${ }^{6}$. Russian scientists' have been evaluating the effects of ecdysterones for years. Oral administration of Leuza (herbal ecdysterone) in male albino mice caused a statistically significant increase in the time of running ${ }^{19}$. After 20 days of supplementation, there was a significant increase in work capacity. The same researchers evaluated the effects of 20-day 
administration of ectisten-containing tincture of leuzea and leveton on humoral immunity of track and field runners for distances of 5,000 and 10,000 m. Intensive cyclic physical activity induced significant decrease of IgG and IgA in blood serum of the athletes. These researchers concluded that both supplements contributed to restoration of the lowered IgG and IgA, while the working capacity of the athletes grew by 10 to $15 \%{ }^{20}$. Furthermore, Chermnykh et al ${ }^{5}$ compared beta-ecdysterone with dianabol which is an extremely powerful anabolic steroid, suggesting both beta-ecdysterone and dianabol increased the size and strength of the muscles. These researchers concluded that ecdysterone had a greater anabolic action on the contractile proteins of the skeletal muscles than dianabol.

The most often cited scientific study on ecdysterone was published in Scientific Sports Bulletin by Simakin ${ }^{21}$. This study sought to determine the effect of ecdysterone on muscle tissue mass and fat mass, while testing for hormonal changes in the subjects. Seventy-eight highly trained male and female athletes served as subjects in one of three experimental groups: protein, protein and ecdysterone, and placebo. Those consuming just protein, showed only a slight increase in muscle mass for the 10 day period of time, while the placebo group experienced a slight reduction in lean muscle. The addition of ecdysterone in conjunction with protein intake resulted in a 6-7\% increase in lean muscle tissue with nearly a $10 \%$ reduction in fat. Finally, Gadzhieva and colleagues ${ }^{22}$ reported that 3-weeks of Ekdisten, leveton, and Prime Plus (combination of Ekdisten and pure protein) supplementation during training increased skinfold determined muscle mass, decreased fat mass, and increased total work during training. Additionally, Ekdisten and Prime Plus supplementation appeared to promote the greatest gains during training. These studies found that ecdysterone might increase work capacity, decrease fat mass, and increase lean muscle mass.

Results of the present study contrast these reports. In this regard, ecdysterone supplementation had no significant effects on body mass, body composition, strength, or markers of anabolic and catabolic status. Since most of the previous studies reporting positive effects of ecdysterones have been reported in obscure journals with limited details available to evaluate the experimental design and quality of the research, it is difficult to compare results. Nevertheless, present findings do not support the purported ergogenic benefit of ecdysterone supplementation in resistancetrained males.

The last supplement investigated was sulfopolysaccharide, which is advertised to bind to myostatin and inhibit its activity in skeletal muscle. Of the three supplements examined in our study, sulfo-polysaccharide supplementation has the best theoretical rationale as a potential ergogenic aid. Myostatin is a cytokine that works by inhibiting the proliferation of satellite cells and the differentiation of myoblasts ${ }^{10}$. Research in mice has shown that binding or blocking myostatin results in dramatic increases in body mass, muscle mass, muscle size, and strength following administration of antibodies that are specific for the cytokine myostatin ${ }^{11,12}$. To date, only one human study has administered sulfopolysaccharides in conjunction with a resistance training protocol ${ }^{13}$. This study examined 12 -weeks of resistance training and cystoseira canariensis supplementation on serum levels of myostatin and muscle strength and body composition in twenty-two untrained males. Training consisted of three days per week using 3 sets of 6 to 8 repetitions at $85-90 \% 1$ RM. The researchers concluded that 12-weeks of heavy resistance training and $1200 \mathrm{mg} / \mathrm{d}$ of cystoseira canariensis supplementation appeared to be ineffective at inhibiting serum myostatin and increasing muscle strength and mass or decreasing fat mass. The explanation for the dramatic effects in animals while no observed effects in humans probably lies in the fact that myostatin specific antibodies were used in the animal model, while human models use sulfo-polysaccharides that are only advertised to bind to myostatin. Results of this study support this prior report in that CSP3 supplementation had no significant effects on body composition, training adaptations, or markers of anabolic/catabolic status in resistance-trained men.

Based on the results of this study, we conclude that supplementation of methoxyisoflavone, ecdysterone, and/or sulfo-polysaccharides (CSP3) do not have any affect on training adaptations and/or anabolic/catabolic status in resistance-trained subjects. These findings contrast marketed claims that these nutrients promote muscle accretion, increase anabolic status, decrease adipose tissue, and/or enhance training adaptations in resistancetrained athletes. These data are representative of a specific training protocol as well as specific prescribed doses of the experimental supplements, but the doses used represent the recommended amount found in these types of supplements. 
Acknowledgements. We would like to thank the subjects that participated in this study and the laboratory assistants in the Exercise \& Sport Nutrition Lab (ESNL) who assisted with data collection and analysis. This study was funded by a research grant from MuscleTech Research \& Development, Inc., (Mississauga, ON) to Baylor University. Researchers in the ESNL independently collected, analyzed and interpreted the results from this study and have no financial interests in the results of this study. Presentation of results in this study does not constitute endorsement by Baylor University or its authors of the supplements investigated.

\section{REFERENCES}

1. Messina, M. and V. Messina, Soyfoods, soybean isoflavones, and bone health: a brief overview. J Ren Nutr, 2000. 10(2): p. 63-8.

2. Feuer L, F.L., Nogradi M, Metabolic 5-methyl-isoflavone-derivatives, process for the preparation thereof and compositions containing the same. United States Patent, 1979(4,163,746).

3. Ohta, H., et al., Effects of 1-year ipriflavone treatment on lumbar bone mineral density and bone metabolic markers in postmenopausal women with low bone mass. Horm Res, 1999. 51(4): p. 178-83.

4. Scheiber, M.D. and R.W. Rebar, Isoflavones and postmenopausal bone health: a viable alternative to estrogen therapy? Menopause, 1999. 6(3): p. 233-41.

5. Chermnykh, N., Shimanovskii, NL, Shutko, GV, Syrov, VN, The action of mehandrostenolone and ecysterone on the physical endurance of animals and on protein metabolism in the skeletal muscles. Farmakol Toksikol, 1988. 51(6): p. 57-60.

6. Sláma K, L.R., Insect hormones - ecdysteroids: their presence and actions in vertebrates. Eur J Entoniol, 1995. 92: p. 355-37.

7. Syrov VN, N.A., Sultanov MB, Action of phytoecdysteroids on the bile-secretory function of the normal liver and in experimental hepatitis. Farmakol Toksikol, 1986. 49: p. 100-103.

8. Syrov, V.N., et al., [Effect of phytoecdysteroids and nerobol on parameters of carbohydrate and lipid metabolism and phospholipid spectrum of liver mitochondrial membrane in experimental diabetes mellitus of rats]. Ukr Biokhim Zh, 1992. 64(4): p. 61-7.

9. Chiang, H.C., J.J. Wang, and R.T. Wu, Immunomodulating effects of the hydrolysis products of formosanin C and beta-ecdysone from Paris formosana Hayata. Anticancer Res, 1992. 12(5): p. 1475-8.

10. Thomas, M., et al., Myostatin, a negative regulator of muscle growth, functions by inhibiting myoblast proliferation. $\mathrm{J}$ Biol Chem, 2000. 275(51): p. 40235-43.

11. Lin, J., et al., Myostatin knockout in mice increases myogenesis and decreases adipogenesis. Biochem Biophys Res Commun, 2002. 291(3): p. 701-6.

12. Bogdanovich, S., et al., Functional improvement of dystrophic muscle by myostatin blockade. Nature, 2002. 420(6914): p. 418-21.

13. Willoughby, D.S., Effects of an alleged myostatin-binding supplement and heavy resistance training on serum myostatin, muscle strength and mass, and body composition. Int J Sport Nutr Exerc Metab, 2004. 14(4): p. 461-72.

14. Nakamura, T., et al., Follistatin, an activin-binding protein, associates with heparan sulfate chains of proteoglycans on follicular granulosa cells. J Biol Chem, 1991. 266(29): p. 19432-7.

15. Kellie, E., Diagnostic and therapeutic technology assessment. Measurement of bone density with dual-energy X-ray absorptiometry (DEXA). Jama, 1992. 267(2): p. 286-8, 290-4.

16. Feuer, L., US Pataent 3949085: Anabolic-weight-gain promoting compositions containing isoflavone derivatives and method using same. 1974.

17. Feuer, L., US4163746: Metabolic 5-methyl-isoflavone-derivatives, process for the preparation thereof and compositions containing the same. 1977.

18. Incledon T, V.G.D., Antonio J, The effects of 5-methyl-7-methoxyisoflavone on body composition and performance in college-aged men. Med Sci Sports Exerc, 2001. 33(5).

19. Azizov AP, S.R., Chubarova AV, The effect of elton, leveton, fitoton and adapton on the work capacity of experimental animals. Eksp Klin Farmakol, 1998. 61(3).

20. Azizov AP, S.R., Chubarova AV, Effects of leuzea tincture and leveton on humoral immunity of athletes. Eksp Klin Farmakol, 1997. 60(60): p. 47-48.

21. Simakin, S.Y., et al., The Combined Use of Ecdisten and the Product 'Bodrost' during Training in Cyclical Types of Sport. Scientific Sports Bulletin, 1988. 2.

22. Gadzhieva, R.M., et al., [A comparative study of the anabolic action of ecdysten, leveton and Prime Plus, preparations of plant origin]. Eksp Klin Farmakol, 1995. 58(5): p. 46-8. 